\title{
Free carrier absorption as a probe of carrier dynamics: A Monte Carlo based study for silicon
}

\author{
Hongtao Jiang, John M. Hinckley, and Jasprit Singh \\ Department of Electrical Engineering and Computer Science, The University of Michigan, \\ Ann Arbor, Michigan 48109-2122
}

(Received 27 December 1996; accepted for publication 2 February 1997)

\begin{abstract}
The Monte Carlo method normally used for transport studies in semiconductors is extended and used to study free carrier absorption of subband gap radiation in semiconductors. The approach is applied to $n$-type silicon where we find very good agreement with experimental results and calculations based on quantum electrodynamics. This method also allows us to study free carrier absorption in semiconductors with a dc bias. With a dc bias, the absorption coefficient has a strong dependence on the polarization of the ac field. We show that the absorption coefficient can be directly related to the carrier temperature as well as the momentum and energy relaxation times. Thus important carrier dynamics information can be obtained from free carrier absorption measurements in the presence of a dc field. (C) 1997 American Institute of Physics.

[S0003-6951(97)01214-X]
\end{abstract}

The absorption processes for subband gap electromagnetic radiation in semiconductors falls into two categories: (i) absorption processes which can only be understood on the basis of a strictly quantum mechanical treatment and require that the initial and final electron states differ by the photon energy (to within the phonon energy). These processes involve multiple bands (or valleys) within the conduction or valence bands; (ii) processes which are forbidden in the lowest order perturbation theory and which can be adequately described by the classical Drude-Zener theory which has been quite successful in explaining free carrier absorption in metals.

In case of $n$-type silicon, it is found experimentally that for radiation with wavelength between 1.5 and $6.0 \mu \mathrm{m}$ absorption is dominated by the first process. For longer wavelength radiation, the absorption coefficient shows a $\alpha \propto \lambda^{2}$ behavior characteristic of the Drude-Zener theory. While the classical theory has been widely used in metals, it has not been exploited in semiconductors. Second order perturbation theory has been used by Fan, Spitzer, and Collins ${ }^{1}$ to calculate free carrier absorption assisted by phonons and impurity scattering. Recently, Huang, Yee, and Soma ${ }^{2}$ have improved the results by using a nonparabolic band. Although these quantum theories give satisfactory results, they do not include carrier-carrier scattering and degeneracy effects which are important at high doping. It is also difficult to extend these approaches to include nonlinear effects arising from very strong radiation fields, or to calculate the absorption in a structure with a high dc bias.

In this article, we address two issues: (i) Can the standard Monte Carlo method used for transport calculations in semiconductors be extended to study free carrier absorption? (ii) Can free carrier absorption in samples with a dc bias be used to probe the carrier dynamics? We address these questions in Si where several careful measurements of free carrier absorption are available in the literature for no applied dc bias. So far there have been no experiments on free carrier absorption in semiconductors with applied dc bias. Our theoretical results show that such measurements would reveal very useful information on carrier dynamics.
We use an ensemble Monte Carlo method to study the transport response of an electron gas in silicon. The scattering processes that are accounted for include inelastic acoustic phonon scattering, optical phonon scattering, intervalley phonon scattering, ionized impurity scattering, and electronelectron scattering. The material parameters used are taken from Refs. 3 and 4. These parameters give good agreement to the usual transport phenomena such as velocity-field curves.

The infrared radiation is represented in a classical picture by a field

$$
F(t)=F_{0} \sin \omega t .
$$

It is well known that the influence of the magnetic field is negligible compared to that of the electric field. The optical power, $I$, gives the field amplitude through the relation

$$
F_{0}^{2}=\frac{2 I}{c n \varepsilon_{0}},
$$

where $c$ is the velocity of light and $n$ is the refractive index.

Using ensemble Monte Carlo techniques, particles are followed in time until energy balance is reached, i.e., the energy loss through scattering equals the energy gain, $E_{\mathrm{abs}}$, from the ac field. The absorption coefficient is then given by

$$
\alpha=\frac{E_{\mathrm{abs}} n_{e}}{I t n_{s}},
$$

where $n_{e}$ is the carrier concentration, $n_{s}$ is the number of particles being simulated, and $t$ is the simulation time.

In Fig. 1, we show results of the calculated free carrier absorption for several doping concentrations in $\mathrm{Si}$. The doping values and compensation ratios used for these results correspond to the experimental cases reported in Ref. 5. We also show in Fig. 1 the experimentally measured results (shown by the solid lines). In this article, we show results for samples 2, 4, and 5 of Ref. 5. These have doping and carrier densities that are well separated. The carrier density and impurity density for sample 2 are estimated in Ref. 5 to be $8.0 \times 10^{16}$ and $9.5 \times 10^{16} \mathrm{~cm}^{-3}$. For sample 4, the values are $3.2 \times 10^{17}$ and $1.26 \times 10^{18} \mathrm{~cm}^{-3}$. For sample 5, they are 6.1 


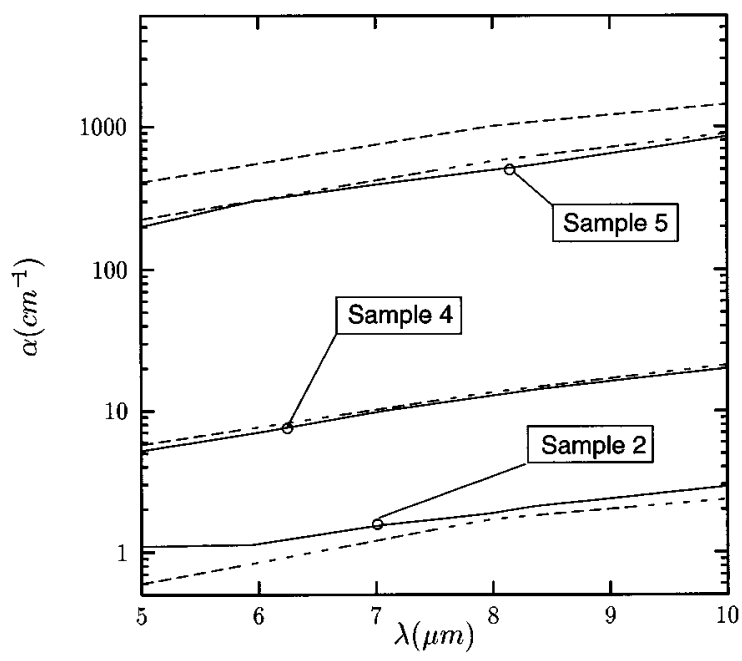

FIG. 1. Absorption coefficient vs wavelength results calculated for a number of samples described in Ref. 5. Also plotted are the experimentally measured results from Ref. 5 (solid line). The free carrier density and impurity density for each sample is given in the text.

$\times 10^{18}$ and $4.6 \times 10^{19} \mathrm{~cm}^{-3}$. We note that the Monte Carlo based formalism gives very good agreement with experiments for samples 4 and 2 . For the heavily doped sample 5, our results (dotted line) are larger than those of the experiment if we use the values for the compensation parameter given in Ref. 5. This may be because of the uncertainty in the method used in Ref. 5 to determine the impurity concentration. At doping densities $N_{i}>10^{19} \mathrm{~cm}^{-3}$, it is difficult to obtain the correct doping density although one can find the free carrier density quite accurately. If we maintain the same carrier density but change the impurity concentration to 2.0 $\times 10^{19} \mathrm{~cm}^{-3}$, we get the results denoted by the dash-dot curve in Fig. 1 which gives a good agreement with experiments.

As noted earlier, several groups have used second order perturbation theory and have also achieved good agreement with experiments. The advantage of the Monte Carlo method arises from the ease with which additional physical effects (additional scattering mechanisms or dc fields) can be included.

In the case of a dc field, the absorption coefficient due to free carriers will be dependent on the polarization of the ac field with respect to the dc field. In order to develop an understanding of how the absorption coefficient depends on the carrier dynamics, we develop a simple model. The results of this model are then compared to the results obtained from the Monte Carlo simulations.

We use semiclassical equations to analyze the free carrier absorption coefficient under dc bias. The equations for the motion of electrons are:

$$
\begin{aligned}
& \frac{d E}{d t}=e \mathbf{v} \mathbf{F}-\frac{\left[E-(3 / 2) k T_{L}\right]}{\tau_{E}}, \\
& \frac{d \mathbf{p}}{d t}=e \mathbf{F}-\frac{\mathbf{p}}{\tau_{m}},
\end{aligned}
$$

where $E, \mathbf{p}, \tau_{E}, \tau_{m}$ are carrier's energy, momentum energy,

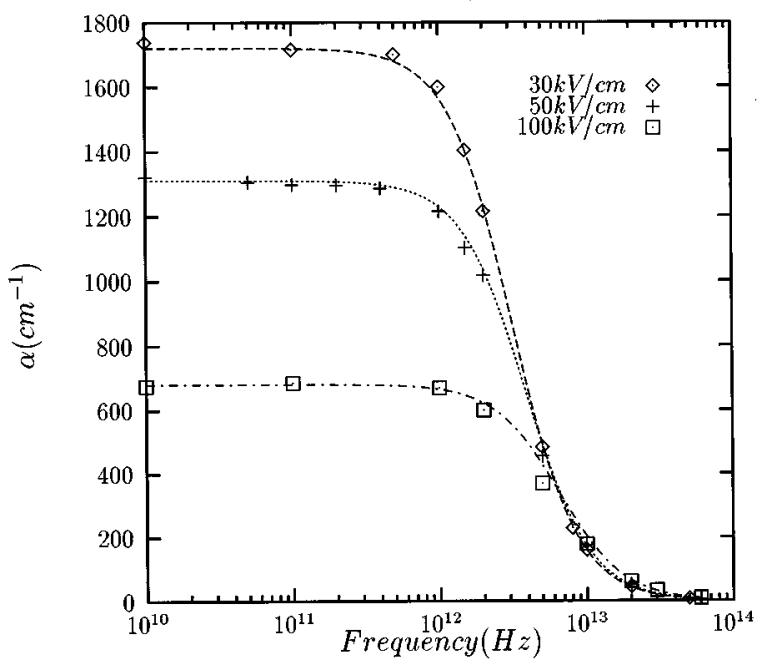

FIG. 2. Absorption coefficient as a function of frequency for $n$-type $\mathrm{Si}$ doped at a carrier density $3.2 \times 10^{17} \mathrm{~cm}^{-3}$ and an impurity concentration $1.26 \times 10^{18} \mathrm{~cm}^{-3}$. The results are shown for the case where laser electric field is normal to the dc field.

and momentum relaxation time, respectively. The electric field is $\mathbf{F}$ while $T_{L}$ denotes the lattice temperature.

We have solved these equations perturbatively treating the ac field effects as a perturbation on the dc field. This allows us to obtain the power absorbed per carrier from the ac field in terms of the parameters $T_{L}, \tau_{m}$, and $\tau_{E}{ }^{6}{ }^{6}$

When the dc field is perpendicular to the polarization of the light, we get

$$
\alpha_{\perp}\left(F_{0}\right)=\mu_{\perp}\left(F_{0}\right) \frac{e n_{e}}{c n \varepsilon_{0}} \frac{1}{1+\left(\omega \tau_{m}\right)^{2}}
$$

where $\mu_{\perp}\left(F_{0}\right)$ is the differential transverse mobility at dc bias $F_{0}$.

When the dc and ac fields are parallel, we get

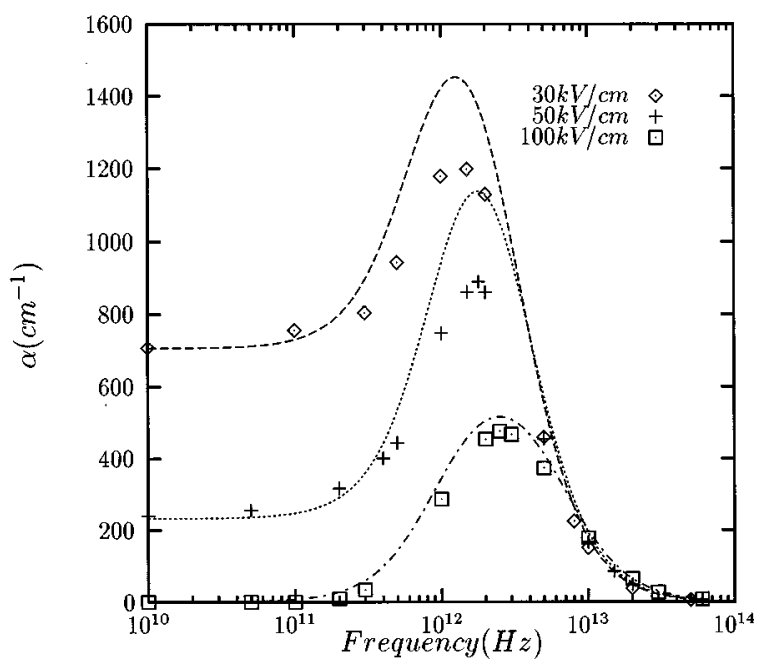

FIG. 3. Absorption coefficient as a function of frequency for $n$-type Si with the same carrier density and impurity concentration as Fig. 2. The results are shown for the case where the laser electric field is parallel to the dc field. 


$$
\begin{aligned}
\alpha_{\|}\left(F_{0}\right)= & \alpha_{\perp}\left(F_{0}\right)\left\{1-\left(1-\frac{3 k T_{L}}{2 E_{0}}\right)\right. \\
& \left.\times \frac{1-\omega^{2} \tau_{m} \tau_{E}\left[1.5+0.5\left(\omega \tau_{m}\right)^{2}\right]}{\left[1+\left(\omega \tau_{m}\right)^{2}\right]\left[1+\left(\omega \tau_{E}\right)^{2}\right]}\right\}
\end{aligned}
$$

and in the hot electrons region $\left(3 k T_{L} / 2 \ll E_{0}\right)$, we get

$$
\alpha_{\|}\left(F_{0}\right)=\alpha_{\perp}\left(F_{0}\right) \frac{\left(\omega \tau_{E}\right)^{2}}{1+\left(\omega \tau_{E}\right)^{2}} .
$$

It is easy to see, $\alpha_{\|}$reaches maximum at $\omega=1 / \sqrt{\tau_{E} \tau_{m}}$.

In Fig. 2, we show absorption coefficient versus frequency for the case where the ac field is perpendicular to the dc field. The results are shown for doping parameters corresponding to those of sample 4 discussed earlier. The dc field values are 30,50 , and $100 \mathrm{kV} / \mathrm{cm}$. The ac field is kept at 4 $\mathrm{kV} / \mathrm{cm}$. We also show fits to the Monte Carlo results using the semiclassical model discussed above. The agreement is quite good. We get the value of the momentum relaxation time from the $\alpha$ vs $f$ curve.

In Fig. 3, we show the $\alpha$ vs $f$ results obtained from Monte Carlo results along with a fit based on our model for the case where the ac field is parallel to the dc field. Once again the agreement between the two is reasonably good. We note that the low frequency $\alpha$ directly gives the carrier temperature as can be seen from Eq. (7) and this value agrees with the value we obtained directly from ensemble Monte Carlo simulation. This curve has an increase in the absorption coefficient when the frequency starts to approach the scattering time. At high frequency, there is the usual roll-off in $\alpha$.

In summary, we find that the Monte Carlo method can be used to fit the experimentally measured $\alpha$ vs $\omega$ data for free carrier absorption. We also find that important carrier dynamics parameters such as carrier energy, momentum, and energy relaxation times can be obtained from the $\alpha$ vs $\omega$ measurements.

This work was funded by the U.S. Army URI program (DAAL03-92-G-0109) and a grant from the U.S. Air Force (Grant No. AFOSR-91-0349).

${ }^{1}$ H. Y. Fan, W. Spitzer, and R. J. Collins, Phys. Rev. 101, 566 (1956).

${ }^{2}$ H. C. Huang, S. Yee, and M. Soma, J. Appl. Phys. 67, 2033 (1989).

${ }^{3}$ C. Jacoboni and L. Reggiani, Rev. Mod. Phys. 55, 645 (1983); The Monte Carlo Method for Semiconductor Device Simulation (Springer, New York, 1989).

${ }^{4}$ K. Hess, Advanced Theory of Semiconductor Devices (Prentice-Hall, Englewood Cliffs, NJ, 1988).

${ }^{5}$ W. Spitzer and H. Y. Fan, Phys. Rev. 108, 268 (1957).

${ }^{6}$ H. Jiang, J. M. Hinckley, and J. Singh (unpublished). 